\title{
Genetic Spectrum and Clinical Features in a Cohort of Chinese Patients with Autosomal Recessive Cerebellar Ataxias
}

\section{Hao-Ling Cheng}

Zhejiang University School of Medicine Second Affiliated Hospital

\section{Ya-Ru Shao}

Zhejiang University School of Medicine Second Affiliated Hospital

\section{Yi Dong}

Zhejiang University School of Medicine Second Affiliated Hospital

\section{Hai-Lin Dong}

Zhejiang University School of Medicine Second Affiliated Hospital

\section{Lu Yang}

Zhejiang University School of Medicine Second Affiliated Hospital

\section{Yin Ma}

Zhejiang University School of Medicine Second Affiliated Hospital

\section{Ying Shen}

Zhejiang University School of Medicine

Zhiying Wu ( $\nabla$ zhiyingwu@zju.edu.cn )

Zhejiang University School of Medicine Second Affiliated Hospital

\section{Research Article}

Keywords: Autosomal recessive cerebellar ataxias, Chinese, Genetic spectrum, Structural variation, Clinical features

Posted Date: June 12th, 2021

DOI: https://doi.org/10.21203/rs.3.rs-576861/v1

License: (c) (i) This work is licensed under a Creative Commons Attribution 4.0 International License. Read Full License 


\section{Abstract}

Background: Although many causative genes have been uncovered in recent years, genetic diagnosis is still missing for approximately $50 \%$ of autosomal recessive cerebellar ataxia (ARCA) patients. Few studies have been performed to determine the genetic spectrum and clinical profiles of ARCA patients in the Chinese population.

Methods: Fifty-four Chinese index patients with unexplained autosomal recessive or sporadic ataxia were investigated by whole-exome sequencing (WES) and copy number variation (CNV) calling with Exome Depth. Likely causal CNV predictions were validated by CNVseq.

Results: Thirty-eight mutations including 29 novel ones were identified in 25 out of 54 patients, providing a $46.3 \%$ positive molecular diagnostic rate. Ten different genes were involved, and the four most common genes were SACS, SYNE1, ADCK3 and SETX, which accounted for $76.0 \%(19 / 25)$ of the positive cases. The de novo microdeletion in SACS was firstly reported in China and the uniparental disomy of $A D C K 3$ was reported for the first time worldwide. Furthermore, the clinical features of the patients carrying SACS, SYNETand ADCK3 mutations were summarized.

Conclusions: Our results expand the genetic spectrum and clinical profiles of ARCA patients, demonstrate the high efficiency and reliability of WES combined CNV analysis in diagnosing suspected ARCA, and emphasize the importance of complete bioinformatics analysis of WES data in making an accurate diagnosis.

\section{Background}

Autosomal recessive cerebellar ataxias (ARCAs) comprise a heterogeneous group of rare metabolic and degenerative genetic disorders that are characterized by progressive damage of the cerebellum and/or its associated afferent tracts $[1,2]$. The overall prevalence of ARCAs is estimated to be $3-5 / 100,000$ in the general population [3], but the prevalence of each individual type may vary among racial and ethnic groups [2,4]. Most ARCAs are early onset before 30 years old, and the major clinical feature is progressive cerebellar ataxia, which is variably combined with dysarthria, ophthalmoplegia, pyramidal and extrapyramidal signs, peripheral neuropathy, cognitive impairment and other symptoms $[1,5,6]$.

With the advanced technologies in genetic screening, more than 90 genes have been identified in ARCAs [6]. Friedreich's ataxia (FRDA) was the most common ARCA, accounting for up to $25 \%$ of all ARCAs, and followed by ataxia-telangiectasia (AT) or ataxia with oculomotor apraxia (AOA) [2]. It is now increasingly acknowledged that spectrin repeat-containing nuclear envelope protein (SYNE1) ataxia and autosomal-recessive spastic ataxia of Charlevoix-Saguenay (ARSACS; gene: SACS), which were long thought to be largely confined to a specific group of French-Canadian populations are relatively frequent ARCAs distribute around the world [2, 7]. Despite the discovery of many disease-causing genes in recent years, the genetic cause of ARCAs remains elusive in more than $50 \%$ of affected individuals $[8,9]$. The advent of whole-exome sequencing (WES) technology not only has enabled the efficient diagnosis with 
single-nucleotide variants (SNVs) and small indels [10], but also the new WES-based method, such as Exome Depth, has successfully detected structural variation (SV) like copy number variations (CNVs) in many hereditary diseases [11]. Moreover, owing to the low incidence, there are few studies reported the rare genetic or clinical characteristic of ARCA patients in the Chinese population.

In this study, we performed WES and CNV calling for 54 unrelated Chinese patients with AR/sporadic hereditary cerebellar ataxia ( $\mathrm{HCA})$ to assess the possibility of detecting mutations with this combined approach and their prevalence. We provide a definitive genetic diagnosis for 25 patients included one patient with large deletion and one with uniparental disomy (UPD), thereby demonstrating that a combined approach of WES and CNV analysis can lead to an estimated diagnostic rate of $46.3 \%$ of ARCA patients. The phenotypes of ARCA patients carrying rare gene mutations were highlighted. Our results expand the genetic spectrum and clinical phenotypes of ARCA patients in the Chinese population.

\section{Materials And Methods}

\section{Subjects}

All patients were consecutively enrolled from Huashan Hospital of Fudan University and the Second Affiliated Hospital of Zhejiang University School of Medicine between August 9, 2008 and May 5, 2021. Patients were evaluated and diagnosed with HCA based on Harding's criteria [12] by at least two senior neurologists. Informed consent was signed by all participants or their guardians. This study was approved by the Ethics Committees of the above two hospitals.

Fifty-two patients enrolled in the study met the following inclusion criteria: (1) progressive cerebellar ataxia; (2) autosomal recessive inheritance with the presence of similar patients in the sibling and/or consanguineous union of the parents or sporadic cases with onset before the age of 40 years; (3) molecular analysis negative for mitochondrial ataxia or 10 common dynamic mutation subtypes of SCA (SCA1, 2, 3, 6, 7, 8, 10, 12, 17 or DRPLA) and Huntington's disease; and (4) exclusion of other identified etiologies (e.g., multiple system atrophy, Niemann-Pick disease, Parkinson's disease, multiple sclerosis, viral infection, alcohol or drug intoxication, or paraneoplastic syndrome). In addition, 1000 unrelated Chinese individuals without a history of ataxia were included as controls.

\section{Genomic DNA extraction and wholeexome sequencing}

Genomic DNA was extracted from peripheral blood samples of all patients by using the QIAamp Blood Genome Extraction Kit (Qiagen, Germany) following a standard protocol. DNA was subjected to wholeexome Illumina sequencing, the detailed protocol for which was given in our previous study [13]. All variants were annotated by ANNOVAR. Two public databases, including the 1000 Genomes Project (http://browser.1000 genomes.org) and Exome Aggregation Consortium (ExAC; http://exac. broadinstitute.org/), and our in-house WES database which containing 1000 Chinese control individuals were used to check the frequency in the general population. Three software programs, including SIFT (http://sift.jcvi.org/), PolyPhen-2 (http://genetics.bwh. harvard.edu/pph2/) and 
Mutation Taster (http://www. mutationtaster.org/), were used to predict the possible deleterious effects of mutations. Moreover, these variants were compared with the Human Gene Mutation Database (HGMD, Professional 2021.1, http://www.hgmd.cf.ac.uk/) to determine whether they were known or novel. Finally, the interpretation and classification of variants was performed based on the American College of Medical Genetics and Genomics (ACMG) standards [14].

\section{Copy number variation analysis}

CNVs from WES read-depth was inferred using Exome depth from the GATK version 4 according to the developers' guidelines [15]. For these analyses, each test exome was compared with a set of matched, aggregate reference samples. CNV calls were annotated using AnnotSV [16]. Candidate CNVs were prioritized by exon number, Bayes factor, minor allele frequency and the ratio of observed/expected number of reads. Candidate CNVs were further proved by the CNVseq, which performed a low-coverage WGS strategy and including the DNA extraction, interruption, library construction and sequencing by Illumina HiSeq 2500 (Illumina, San Diego, United States). The databases ISCA, DGV, Decipher, OMIM, ClinVar and ClinGen were used to analyze the CNVs. Comprehensive assessments of CNV hazard levels were undertaken based on a frequency database and annotation information according to the ACMG standards and guidelines [17].

\section{Affymetrix CytoScan® Dx Assay}

The Affymetrix CytoScan® Dx Assay was used to identify the UPD, which utilizes a high-density combined SNP and comparative genomic hybridization (CGH) array platform, which assesses approximately 2,696,550 markers, including approximately 750,000 SNP markers [18]. The wholegenome screening and analysis of chromosomal rearrangements by Affymetrix CytoScan $\circledast$ Dx Assay was performed according to the manufacturers' recommendations [18].

\section{Sanger sequencing and parent analysis}

After analyzing and filtering the WES data, Sanger sequencing was performed to confirm the sequencing results and family co-segregation pattern. The parenthood of patients with de novo variants were analyzed using 21 core short tandem repeat (STR) regions, including D19S433, D5S818, D21S11, D18S51, D6S1043, AMEL, D3S1358, D13S317, D7S820, D16S539, CSF1PO, Penta D, D2S441, vWA, D8S1179, TPOX, Penta E, TH01, D12S391, D2S1338, and FGA.

\section{Results}

\section{Twenty-six novel variants were identified in 25 unrelated ARCA patients}

Totally, 38 variants (Table 1) including 29 novel variants and 9 known ones, in 10 genes, were identified in 25 out of 54 ARCA patients. All novel variants (chromatograms were shown in Fig.

1A except chr13:23490196-24866656del) were absent from or present at extremely low frequency in both public databases our in-house WES database. The pathogenicity of variants is consistently predicted by 
different in silico prediction programs. All novel missense variants are highly conserved among animal species (Fig. 1B). According to the ACMG standard, 17 out of 26 novel variants were classified as pathogenic variants, 11 as likely pathogenic variants and the remaining two $(A D C K 3$ : p.R271H, SETX: p.Y2455C) as variants of uncertain significance. Six out of 9 known variants (Additional file 1:

Supplementary Figure 1A) were identified as pathogenic variants and three as likely pathogenic variants.

Therefore, 25 out of 54 (46.3\%) patients were genetically diagnosed with ARCA. Previously, we reported an index patient with AOA2 [19] and an index patient with SCAR27 [20]. Taken together, genetic diagnoses were made for a total of 27 out of $56(48.2 \%)$ ARCA index patients in our center. The most common subtype was ARSACS (22.2\%), followed by SCAR8 (18.5\%), SCAR9 (18.5\%) and AOA2 (14.8\%)

(Fig. 1C). The clinical features of the patients identified with the genetic variants are listed in Table 2.

\section{Identification of a de novo microdeletion and clinical features of patients with SACS mutations}

After WES analysis, a novel homozygous mutation (p.K3646fs) was identified in case 1 without family history (Table 2). However, the heterozygous p.K3646fs was only confirmed in his mother and brother but not in his father after the family segregation. The parent-child relationship was also established by parenthood analysis (Fig. 2A). Therefore, the CNV analysis with Exome Depth was conducted in the proband and a large deletion was detected in chromosome 13. Then, a trio-copy number variation sequencing (CNVseq) was performed and identified a $1.38 \mathrm{Mb}$ deletion (chr13:23490196$24866656 \mathrm{del}$ ) in the proband, but the corresponding chromosome in the parents was normal, which means it was a de novo large deletion (Fig. 2B). The chr13:23490196-24866656 in Genome browser includes the entire SACS gene and 5 other genes (Fig. 2C). Thus, a total of 9 mutations including 8 novel ones in SACS were identified in 6 patients (Table 2).

The mean age of disease onset was 9.5 years (1-39 years), and all onset with gait disturbance was accompanied by sensorimotor neuropathy. Weakness of limbs was present in 4 cases, skeletal abnormality in 5 cases (pes cavus in 4 cases, Fig. 3A and 1 accompanied by flexion deformity of fingers Fig. 3B, dental abnormalities in 1 case, Fig. 3C), listening loss and spasticity in 2 cases, and mental retardation in 1 case. Radiological evaluation of the 3-year-old patient (case 1) showed a normal contract, whereas the other 5 cases showed cerebellum atrophy, thinning of the corpus callosum, bulky pons, bilateral pontine linear hypointense lesions and hyperintensities around the thalamus (Fig. 3D3F), and one had craniocerebral dysplasia (case 4). A characteristic retinal finding of case 2 and case 4 was the presence of yellow streaks of hypermyelinated fibers radiating from the edges of the optic disc and retinal nerve fiber hypertrophy, as demonstrated on ocular coherence tomography (Fig. 3G-3K).

\section{Characteristics of patients with SYNE1 mutations}

Among the 9 novel SYNE1 pathogenic variants found in 5 cases, 3 were frameshift, 4 were nonsense and 2 were splicing variants. The onset age was before 25 years old in 4 patients and at 53 years (late onset) in one patient. In 5 cases, all onset with gait disturbance, 3 had dysarthria and horizontal nystagmus. Case 7 is the earliest onset one with most complex phenotypes including psychiatric symptoms, external 
ophthalmoplegia and myoclonic jerks (Additional file 2: Supplementary Video 1). Case 8 with a homozygous mutation (p.E3053fs) appeared to be pure cerebellar ataxia. Both case 9 and case 11 had sensorimotor neuropathy, and case 9 was also accompanied by tremor, dizziness and pes cavus. Case 10 had a 15-year history of sensorineural hearing loss and mild ataxia. She also exhibited two pathogenic mutations (Additional file 1: Supplementary Figure 1B) including c.6149-3T>G and c.1898dupA (p.E633fs) in PTPRQ (NM_001145026.1). After pedigree verification, her brother affected by impaired hearing but without ataxia, was found to carry these two mutations in PTPRQ but only one heterozygous mutation in SYNE1, and all mutations in SYNE1 and PTPRQ were derived independently from two parents with normal phenotypes (Additional file 1: Supplementary Figure 1C). Owing to the lack of MRI report of case 8 , the remaining three probands were estimated to have a variable degree of cerebellar atrophy.

\section{Identification of a rare uniparental disomy and clinical features of patients with $A D C K 3$ mutations}

After WES analysis, 6 ADCK3 mutations included 3 novel ones (p.R271H, p.L320fs and p.R598H) were identified in 4 males with no family history of disease and one female with an affected sister. The homozygous variant (p.R410X) within ADCK3 was identified in case 10 (Table 2). However, the heterozygous p.R410X was only confirmed in his mother but not in his father, and the parental analysis was normal (Fig. 4A). The CNV analysis of the index patient was normal, too. Thus, the uniparental disomy was considered in this situation. The results of Affymetrix CytoScan ${ }^{\circledR}$ Dx Assay analyses were as follows: arr [hg19] 1pterp36.11 (888,658-25, 445, 510) hmz; arr [hg19] 1q42.12qter (225, 105, 798-249, $198,164) \mathrm{hmz}$, which suggested that the index patient had maternal UPD for a segment of chromosome 1 (Fig. 4B) which included the whole $A D C K 3$ gene (Fig. 4C).

The mean age at onset was 16.2 years and ranged from 2 to 32 years. All the patients showed ataxia signs, and four also revealed dysarthria. Cases 12, 13 and 16 had onset with gait disturbance, case 12 displayed pure ataxia, but cases 13 and 16 had additional cognitive impair. In cases 14 and 15, onset presented with uncontrollable hand shaking, followed later by head shaking. Both were identified to have the p.S616fs mutation, but it was heterozygous in case 14 and homozygous in case 15 . Case 15 had additional dysphagia and SMN. All 5 patients with MR had cerebellar atrophy, and one also had atrophy of the brain stem. Case 15 is a member of the Dong minority group and had started supplementation with $300 \mathrm{mg} /$ day of CoQ10. At 1-year follow-up, he showed clinical improvement, which was more evident in the tremor and gait items. His total SARA scores were 10 at baseline and 4.5 after 1 year; similarly, his ICARS score decreased from 21 to 9 , and his non-ataxia score decreased from 4 to 1 (Additional file 3: Supplementary Table 1). A homozygous mutation was identified by Sanger sequencing in his 24-year-old sister, who had no clinical symptoms yet.

\section{Discussion}

To date, few studies have been conducted to investigate all causative genes and the clinical features for ARCA in the Chinese population. In this study, the prevalence of ARCA was systematically investigated in 54 unrelated AR/sporadic ataxia patients by WES analysis and CNV calling. Thirty-eight 
mutations, including 29 novel mutations in 10 genes related to ARCA, were identified in 25 unrelated patients, leading to a positive molecular diagnosis rate of $46.3 \%$. The de novo microdeletion in SACS was firstly reported in the Chinese population and the UPD of $A D C K 3$ was firstly reported worldwide.

Here, we demonstrate that ARSACS (gene: SACS, n=6, 22.2\%), SCAR8 (gene: SYNE1; n=5, 18.5\%), SCAR9 (gene: $A D C K 3 ; \mathrm{n}=5,18.5 \%$ ) and $A O A 2$ (gene: $S E T X ; \mathrm{n}=4,14.8 \%$ ) are most common recessive ataxia in the Chinese population. FRDA was reported as the most frequent ARCA in Caucasians but was much rare in Chinese [21], thus the identification of dynamic mutations was not undertaken in our study. SACS and SYNE1 mutations have been observed mainly in Quebec and Canada, where ARSACS and SCAR8 are the second and third most common hereditary ataxia [7]. Thus, despite the aetiology of ARCA in Chinese is different from the reported patterns in Caucasians which presumably based on their different genetic background and the ethnic differences, there are still some similarities which may contribute to a better understanding of the epidemiology and mechanism of ARCA.

In total, 14 ARSACS patients from 10 families have been reported in China (Additional file 4:

Supplementary Table 2), including the 5 probands in this study confirmed that nonsense or frameshift mutations in SACS were the most common genetic cause in Chinese patients. In our study, majority of those patients who had at least one truncation variant appeared to have a typical ARSACS clinical presentation with childhood onset of symptoms. But one patient who harbored a homozygous nonframeshift deletion variant (p.3758_3759del), exhibited an atypical disease presentation with an absence of spasticity or pyramidal signs and onset in adulthood, and this same variant was reported in two heterozygous patients, both with early onset (1 and 13 years old) and typical triad symptoms [22, 23]. Thus, whether the truncation variant in SACS is linked to a typical clinical manifestation of ARSACS is an issue that requires further exploration. Even this de novo large deletion containing SACS was the second report in the world [24], the CNV in SACS were already reported in many populations including Belgian, French, Italian, Canadian, German and Chinese [24-30]. Thus, SV might not be extremely rare, and we suggested that tools for detecting SVs such as CNVs should be used routinely for NGS data analysis in order to increase the rate of positive diagnosis.

Defects in SYNE1 are associated with adult-onset, slowly progressive, relatively pure cerebellar ataxia with only a few extracerebellar symptoms (SCAR8), and almost all reported variants that cause this phenotype are protein truncations [7]. Previous studies showed that SYNE1 ataxia accounted for $5.3 \%$ (23/434), $6 \%$ (7/116), and $10.26 \%$ (4/39) of recessive and sporadic ataxia patients in two European combined and one Brazilian cohort [7, 31, 32]. In this study, 9.3\% (5/54) ataxia patients had biallelic truncating variants in SYNE1, demonstrating that SCAR8 is also a common recessive ataxia in China. Because the hearing loss in case 8 was caused by mutations in PTPRQ, the phenotype of two SCAR8 patients (cases 8 and 10) was pure cerebellar ataxia. Moreover, the remaining 3 patients exhibited variable additional extracerebellar with neurological symptoms (peripheral polyneuropathy, mental retardation, dizziness, pes cavus, external ophthalmoplegia, myoclonic jerks) and non-neurologic dysfunctions (psychiatric symptoms). The reported 9 SCAR8 Chinese patients included three presented with pure cerebellar ataxia and 
6 presented with variable ataxia syndrome [33-35]. Thus, in the Chinese population, pure cerebellar ataxia only accounts for $35.7 \%(5 / 14)$ of SCAR8 cases, while the other $64.3 \%(9 / 14)$ of patients show complex ataxia phenotypes with a wide range of noncerebellar abnormalities. It further supports the concept that SYNE1 ataxia is a multisystemic neurodegenerative disease, as proposed by Synofzik et al. in 2016 [7].

A total of 65 pathogenic mutations in $A D C K 3$ have been reported around the world (HGMD, Professional 2021.1), and SCAR9 has also been reported as a common recessive ataxia [36], but rare case of SCAR9 has been reported among the Chinese population before [37]. This study is the first report demonstrated that SCAR9 also had a high frequency in China. The homozygous p.S616fs in ADCK3 has been reported in two siblings from a consanguineous family of Pakistani origin, and both siblings presented with cerebellar ataxia, myoclonus, tremor and dysarthria at age of 10 and 14, respectively [38]. However, in our study, two patients harbored p.S616fs in heterozygous or homozygous form both presented with prominent tremor and mild ataxia symptoms, but onset at adolescence and adulthood, respectively. Moreover, the homozygous patient presented with additional dysphagia and peripheral neuropathy, and his sister was identified to have the mutation but was still asymptomatic at the age of 24 . Thus, the clinical presentation of SCAR9 may be highly variable, even in patients with the same mutation from one family. Our study also proved that supplementation with COQ10 was significantly helpful for SCAR9 patients, even though this therapy had different curative effects in several studies [38-40]. The UPD of $A D C K 3$ identified in our study is the first reported worldwide, not only enriched the genotypic spectrum of SCAR9, but also emphasized the importance of a detailed analysis of the family segregation.

The absences of diagnosis for 29 patients in the cohort may be induced by the following reasons. First, exome capture is difficult to fully cover all coding regions of the genome, especially regions rich with GC. Second, large genomic rearrangements and trinucleotide expansions cannot be reliably detected from exome-capture data, even though there are some detecting CNVs tools developed and based on the readdepth of NGS data, such as Exome Depth used in this study, cannot easily detect inversion or translocation [41]. Third, it is also likely that some causal variants will outside the coding regions and adjacent splice sites [42]. Finally, the insight into the functional consequences of the variant is missing, such as some synonymous mutations may be causative, too [43]. Some of these issues can be addressed by whole genome sequencing, which companied by substantial additional cost and complex bioinformatics analyses. However, mutations in yet unknown genes of ARCA may play a key role in these unclear disorders.

\section{Conclusions}

In summary, we identified 38 mutations including 29 novel ones in 25 unrelated Chinese ARCA patients. We reported the first UPD of $A D C K 3$ in the world and the first de novo microdeletion of SACS in China. Our results expand the genetic spectrum and clinical profiles of ARCA patients, demonstrate the high efficiency and reliability of WES combined CNV analysis in diagnosing suspected ARCA, and emphasize the importance of complete bioinformatics analysis of WES data in making an accurate diagnosis. 


\section{List Of Abbreviations}

ACMG: American College of Medical Genetics and Genomics; AOA: ataxia with oculomotor apraxia; ARCA: autosomal recessive cerebellar ataxia; ARSACS: autosomal-recessive spastic ataxia of CharlevoixSaguenay; AT: ataxia-telangiectasia; CGH: comparative genomic hybridization; CNV: copy number variation; ExAC: Exome Aggregation Consortium; FRDA: Friedreich's ataxia; HCA: hereditary cerebellar ataxia; HGMD: Human Gene Mutation Database; SNV: single-nucleotide variants; STR: short tandem repeat; SV: structural variation ; SYNE1: spectrin repeat-containing nuclear envelope protein; WES: wholeexome sequencing; UPD: uniparental disomy.

\section{Declarations}

\section{Ethics approval and consent to participate}

The study was approved by the Ethics Committee of the Second Affiliated Hospital of Zhejiang University School of Medicine (protocol code 2015-048, 2015-08-11). And written informed consents for the study have been obtained from all the participants or their legal guardians.

\section{Consent for publication}

When included the individual person's data, consent for publication have been obtained from those people or their legal guardians.

\section{Availability of data and materials}

The data presented in this study are available on request from the corresponding author. The data are not publicly available due to privacy reasons.

\section{Competing interests}

The authors declare that they have no competing interests

\section{Funding}

This work was supported by the grant (82071260, Beijing) to Zhi-Ying Wu from the National Natural Science Foundation, and the research foundation for distinguished scholar of Zhejiang University to ZhiYing Wu (188020-193810101/089, Hangzhou).

\section{Authors' contributions}

Hao-Ling Cheng: Methodology, Data curation, Formal analysis, Supervision, Writing-original draft preparation. Ya-Ru Shao: Methodology, Data curation. Yi Dong: Formal analysis, Supervision. Hai-Lin Dong: Formal analysis, Supervision. Lu Yang: Data curation, Formal analysis. Yin Ma: Methodology. Ying 
Shen: Formal analysis, Supervision. Zhi-Ying Wu: Conceptualization, Resources, Data curation, Formal analysis, Supervision, Writing-Reviewing and Editing. All authors read and approved the final manuscript.

\section{Acknowledgements}

We would like to thank all participants for their support and willingness to participate in this study.

\section{References}

1. Anheim M, Tranchant C, Koenig M. The autosomal recessive cerebellar ataxias. N Engl J Med. 2012;366(7):636-46.

2. Synofzik M, Puccio H, Mochel F, Schols L. Autosomal Recessive Cerebellar Ataxias: Paving the Way toward Targeted Molecular Therapies. Neuron. 2019;101(4):560-83.

3. Manto M, Gandini J, Feil K, Strupp M. Cerebellar ataxias: an update. Curr Opin Neurol. 2020;33(1):150-60.

4. Anheim M, Fleury M, Monga B, et al. Epidemiological, clinical, paraclinical and molecular study of a cohort of 102 patients affected with autosomal recessive progressive cerebellar ataxia from Alsace, Eastern France: implications for clinical management. Neurogenetics. 2010;11(1):1-12.

5. Coutelier M, Hammer MB, Stevanin G, et al. Efficacy of Exome-Targeted Capture Sequencing to Detect Mutations in Known Cerebellar Ataxia Genes. Jama Neurol. 2018;75(5):591-9.

6. Rossi M, Anheim M, Durr A, et al. The genetic nomenclature of recessive cerebellar ataxias. Movement Disord. 2018;33(7):1056-76.

7. Synofzik M, Smets $\mathrm{K}$, Mallaret $\mathrm{M}$, et al. SYNE1 ataxia is a common recessive ataxia with major noncerebellar features: a large multi-centre study. Brain. 2016;139(Pt 5):1378-93.

8. Mallaret M, Renaud M, Redin C, et al. Validation of a clinical practice-based algorithm for the diagnosis of autosomal recessive cerebellar ataxias based on NGS identified cases. J Neurol. 2016;263(7):1314-22.

9. Parodi L, Coarelli G, Stevanin G, Brice A, Durr A. Hereditary ataxias and paraparesias. Curr Opin Neurol. 2018;31(4):462-71.

10. Chen CX, Dong HL, Wei Q, et al. Genetic spectrum and clinical profiles in a southeast Chinese cohort of Charcot-Marie-Tooth disease. Clin Genet. 2019;96(5):439-48.

11. Erin Z, Benyam K, M PE, et al. Copy-number variation contributes $9 \%$ of pathogenicity in the inherited retinal degenerations. Genet Med. 2020;22(6):1079-87.

12. AE H. Clinical features and classification of inherited ataxias, 1993:1-14. 
13. Dong HL, Wei Q, Li JQ, et al. Genetic spectrum of MCM3AP and its relationship with phenotype of Charcot-Marie-Tooth disease. J Peripher Nerv Syst. 2020;25(2):107-11.

14. Richards S, Aziz N, Bale S, et al. Standards and guidelines for the interpretation of sequence variants: a joint consensus recommendation of the American College of Medical Genetics and Genomics and the Association for Molecular Pathology. Genet Med. 2015;17(5):405-23.

15. Plagnol V, Curtis J, Epstein $M$, et al. A robust model for read count data in exome sequencing experiments and implications for copy number variant calling. Bioinformatics. 2012;28(21):2747-54.

16. Geoffroy $\mathrm{V}$, Herenger $\mathrm{Y}$, Kress $\mathrm{A}$, et al. AnnotSV: an integrated tool for structural variations annotation. Bioinformatics. 2018;34(20):3572-4.

17. Riggs ER, Andersen EF, Cherry AM, et al. Technical standards for the interpretation and reporting of constitutional copy-number variants: a joint consensus recommendation of the American College of Medical Genetics and Genomics (ACMG) and the Clinical Genome Resource (ClinGen). Genet Med. 2019;22(2):245-57.

18. Webb BD, Scharf RJ, Spear EA, Edelmann LJ, Stroustrup A. Evaluation of the Affymetrix CytoScan ${ }^{\circledR}$ Dx Assay for developmental delay. Expert Rev Mol Diagn. 2015;15(2):185-92.

19. Lu C, Zheng YC, Dong Y, Li HF. Identification of novel senataxin mutations in Chinese patients with autosomal recessive cerebellar ataxias by targeted next-generation sequencing. Bmc Neurol. 2016;16(1):179.

20. Dong $\mathrm{H}$, Cheng $\mathrm{H}$, Bai $\mathrm{G}$, Shen $\mathrm{Y}$, Wu Z. Novel GDAP2 pathogenic variants cause autosomal recessive spinocerebellar ataxia-27 (SCAR27) in a Chinese family. Brain. 2020;143(6):e50.

21. Zeng J, Wang J, Zeng S, et al. Friedreich's Ataxia (FRDA) is an extremely rare cause of autosomal recessive ataxia in Chinese Han population. J Neurol Sci. 2015;351(1-2):124-6.

22. Rezende Filho FM, Parkinson MH, Pedroso JL, et al. Clinical, ophthalmological, imaging and genetic features in Brazilian patients with ARSACS. Parkinsonism Relat D. 2019;62:148-55.

23. Sun W, Yu M, Zhuo YJ, Wang ZX, Yuan Y. Novel spastic ataxia of Charlevoix-Saguenay gene compound heterozygous mutations in late onset autosomal recessive spastic ataxia of CharlevoixSaguenay. Chin J Neurol. 2017;50(11):831-6.

24. Breckpot J, Takiyama Y, Thienpont B, et al. A novel genomic disorder: a deletion of the SACS gene leading to spastic ataxia of Charlevoix-Saguenay. Eur J Hum Genet. 2008;16(9):1050-4.

25. Miressi F, Faye P, Pyromali I, et al. A mutation can hide another one: Think Structural Variants! Comput Struct Biotec. 2020;18:2095-9. 
26. Terracciano A, Casali C, Grieco GS, et al. An inherited large-scale rearrangement in SACS associated with spastic ataxia and hearing loss. Neurogenetics. 2009;10(2):151-5.

27. Prodi E, Grisoli M, Panzeri M, et al. Supratentorial and pontine MRI abnormalities characterize recessive spastic ataxia of Charlevoix-Saguenay. A comprehensive study of an Italian series. Eur $\mathrm{J}$ Neurol. 2013;20(1):138-46.

28. Dougherty SC, Harper A, Al Saif H, Vorona G, Haines SR. A Chromosomal Deletion and New Frameshift Mutation Cause ARSACS in an African-American. Front Neurol. 2018;9:956.

29. Vill K, Müller-Felber W, Gläser D, et al. SACS variants are a relevant cause of autosomal recessive hereditary motor and sensory neuropathy. Hum Genet. 2018;137(11-12):911-9.

30. Liu L, Li XB, Zi XH, et al. A novel hemizygous SACS mutation identified by whole exome sequencing and SNP array analysis in a Chinese ARSACS patient. J Neurol Sci. 2016;362:111-4.

31. Mademan I, Harmuth F, Giordano I, et al. Multisystemic SYNE1 ataxia: confirming the high frequency and extending the mutational and phenotypic spectrum. Brain. 2016;139(8):e46.

32. Gama MTD, Houle G, Noreau A, et al. SYNE1 mutations cause autosomal-recessive ataxia with retained reflexes in Brazilian patients. Movement Disord. 2016;31(11):1754-6.

33. Guan RY, Wu JJ, Ding ZT, Wang J, Sun YM. Clinical and genetic findings in a cohort of Chinese patients with autosomal recessive spinocerebellar ataxia. Clin Genet. 2020;97(3):532-5.

34. Peng Y, Ye W, Chen Z, et al. Identifying SYNE1 Ataxia With Novel Mutations in a Chinese Population. Front Neurol. 2018;9:1111.

35. Duan X, Hao Y, Cao Z, et al. Autosomal Recessive Cerebellar Ataxia Type 1: Phenotypic and Genetic Correlation in a Cohort of Chinese Patients with SYNE1 Variants. The Cerebellum. 2020.

36. Sun M, Johnson AK, Nelakuditi V, et al. Targeted exome analysis identifies the genetic basis of disease in over $50 \%$ of patients with a wide range of ataxia-related phenotypes. Genet Med. 2019;21(1):195-206.

37. Liu G, Ma D, Li J, et al. A novel COQ8A missense variant associated with a mild form of primary coenzyme Q10 deficiency type 4. Clin Biochem. 2020;84:93-8.

38. Liu YT, Hersheson J, Plagnol V, et al. Autosomal-recessive cerebellar ataxia caused by a novel ADCK3 mutation that elongates the protein: clinical, genetic and biochemical characterisation. Journal of Neurology, Neurosurgery \& Psychiatry. 2014;85(5):493-8.

39. Chang A, Ruiz Lopez M, Slow E, Tarnopolsky M, Lang AE, Munhoz RP. ADCK3-related Coenzyme Q10 Deficiency: A Potentially Treatable Genetic Disease. Movement Disorders Clinical Practice. 2018;5(6):635- 
40. Horvath R, Czermin B, Gulati S, et al. Adult-onset cerebellar ataxia due to mutations inCABC1/ADCK3. Journal of Neurology, Neurosurgery \& Psychiatry. 2012;83(2):174-8.

41. Miressi F, Faye P, Pyromali I, et al. A mutation can hide another one: Think Structural Variants!. Comput Struct Biotec. 2020;18:2095-9.

42. Dong Y, Ni W, Chen WJ, et al. Spectrum and Classification of ATP7B Variants in a Large Cohort of Chinese Patients with Wilson's Disease Guides Genetic Diagnosis. Theranostics. 2016;6(5):638-49.

43. Hunt RC, Simhadri VL, landoli M, Sauna ZE, Kimchi-Sarfaty C. Exposing synonymous mutations. Trends Genet. 2014;30(7):308-21.

\section{Figures}



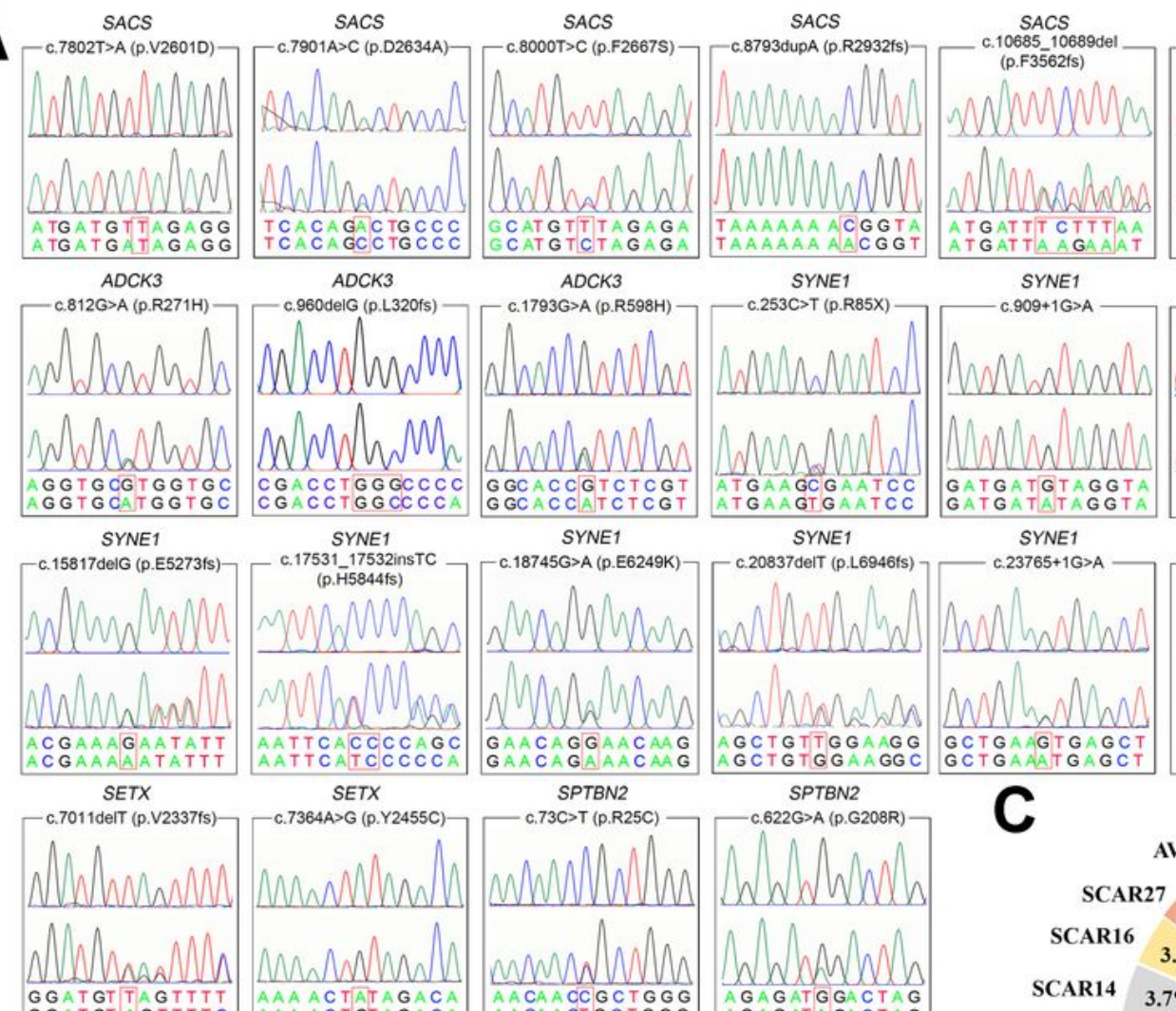

GGATGT AGTTTTC AAA ACTGTAGACA [ACAACTGCTGGG [AGAGATAGACTAG
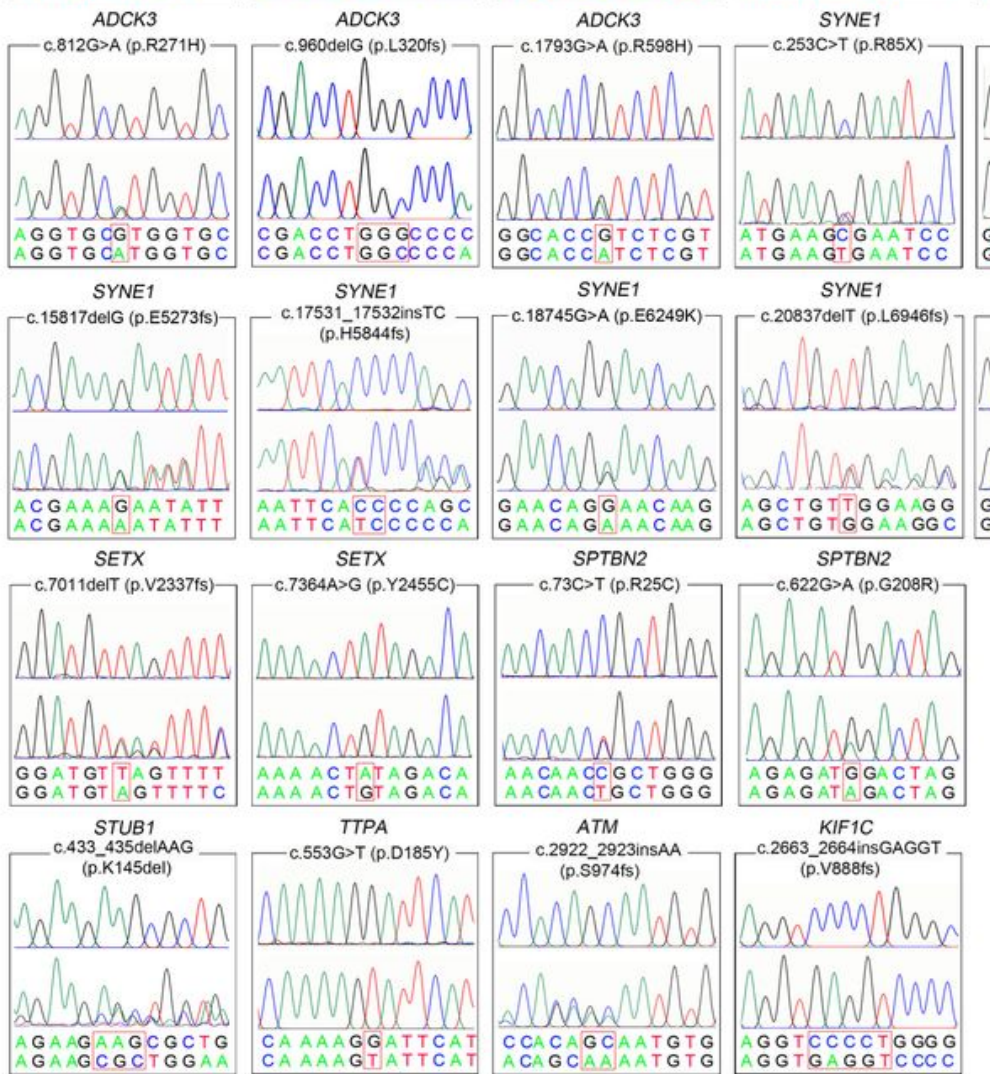

SYNE1

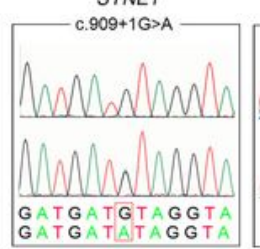

SYNE1

GATGATATAGGT
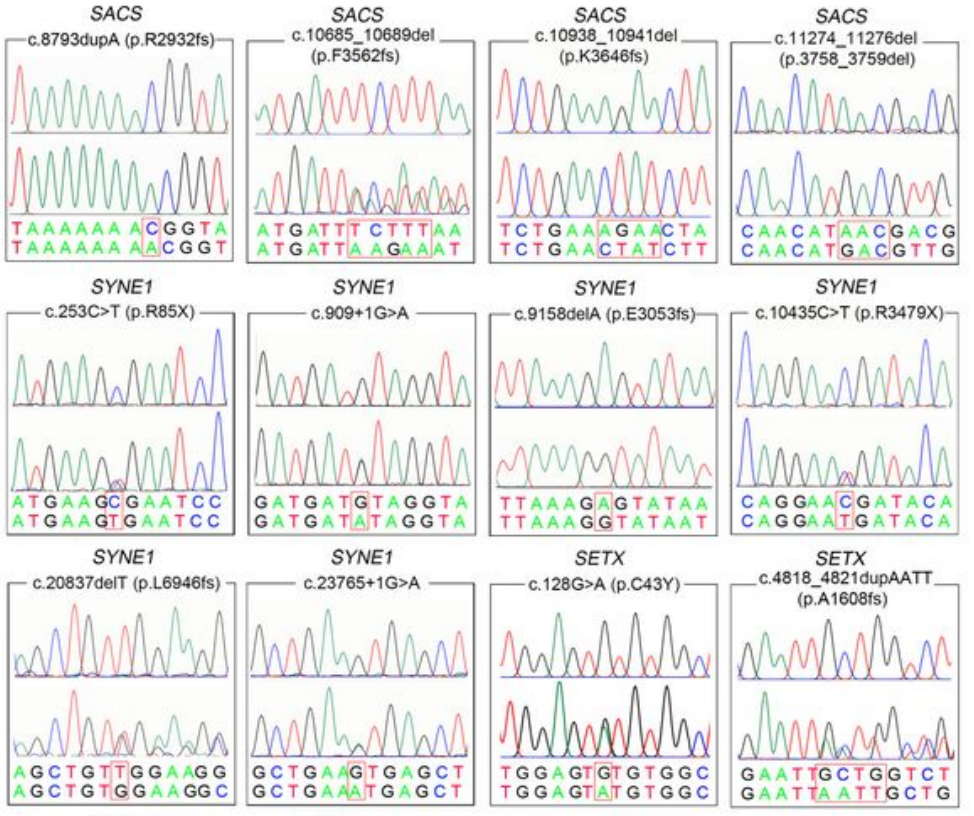

C

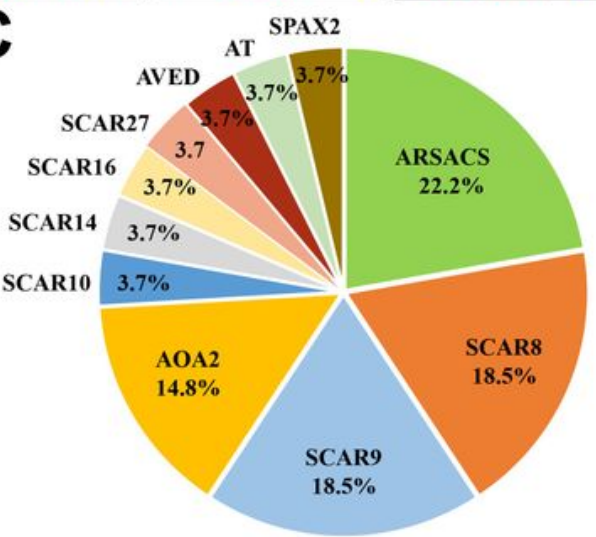

B

$\begin{array}{lcr}\text { Human } & \text { SACS_V2601D } & \text { SACS_D2634A } \\ \text { Chimpanzee } & \text { TEDDVRGIQ } & \text { YHITDCPSF } \\ \text { Monkey } & \text { TEDDVRGIQ } & \text { YHITDCPSF } \\ \text { Dog } & \text { TEDDVRGIQ } & \text { YHITDCPSF } \\ \text { Rat } & \text { TEDDVRGIQ } & \text { YHITDCPSF } \\ \text { Mouse } & \text { TEDDVRGIQ } & \text { YHITDCPSF } \\ \text { Cow } & \text { TEDDVRGIQ } & \text { YHITDCPSF } \\ \text { Chicken } & \text { TEDDVRGIQ } & \text { YHITDCPSF } \\ & \text { TEDDIRGIQ } & \text { YHITDCPSF } \\ & \text { SYNE1_R3479X } & \text { SETX_C43Y } \\ \text { Human } & \text { AIQERAKEA } & \text { YCLECVAEY } \\ \text { Chimpanzee } & \text { AIQERAKEA } & \text { YCLECVAEY } \\ \text { Monkey } & \text { AIQERAKEA } & \text { YCLECVAEY } \\ \text { Dog } & \text { TIKERAKEA } & \text { YCLECNTEY } \\ \text { Rat } & \text { AIQERAKEA } & \text { YCLECVAEY } \\ \text { Mouse } & \text { AIQERAKEA } & \text { YCLECVAEY } \\ \text { Cow } & \text { AIRERTKEA } & \text { YCLECVAEY } \\ \text { Chicken } & \text { LLKDRTREA } & \text { YCLECVVEY }\end{array}$

SACS_F2667S

PGRMFRDLD

PGRMFRDLD

PGRMFRDLD

PGRMFRDLD

PGRMFRDLD

PGRMFRDLD

PGRMFRDLD

PGRMFRDLD

SETX_Y2455C

CDKNYRHDA

CDKNYRHDA

CDKNYRHDA

CDKNYRHDA

CDPNYRHDA

SDPNYRHDA

CDKNYRHDA

SDKSYKKDA
ADCK3_R271H

LCKVRGAAL

LCKVRGAAL

LCKVRGAAL

LCKVRGAAL

LCKVRGAAL

LCKVRGAAL

LCKVRGAAL

LCKVRGAAL

SPTBN2_R25C

DINNRWDLP

DINNRWDLP

DINNRWDLP

DINNRWDLP

DINNRWDLP

DINNRWDLP

DINNRWDLP

DINNRWDLP
ADCK3_R598H

MLRHRLVPP

MLRHRLVPP

MLRHRLVPP

MLKHRLVPP

MLKHR_I PP

MLKHR_I PP

MLKHR VPP

MLKHR_VPP

\section{SPTBN2_G208R}

SWRDGLAFN

SWRDGLAFN

SWRDGLAFN

SWRDGLAFN

SWRDGLAFN

SWRDGLAFN

SWRDGLAFN

SWRDGLAFN
SYNE1_R85X

RRMKRIHAV

RRMKRIHAV

RRMKR IHAV

RRMKRIHAV

RRMKRIIHAV

RRMKRIHAV

RRMKRIHAV

RHL KRIHWV

\section{TTPA_D185Y}

AVLTDSFPL

AVLTDSFPL

AVLTDSFPL

AVLTDSFPL

AVVTDSFPL

AVLTDSFPL

AVLTDSFPL

AVLTDSFPL
SYNE1_K1094X

ELCVIKLPVR ELCYKLPVR ELCVKLPVR ELCVKLPVR ELCVKLPVR ELCVKLPVR ELCVKLPVR ELCVKLPVR

\section{Figure 1}

Chromatogram, homology comparison, and genetic spectrum. A: Chromatograms of 28 novel variants. The upper chromatogram each frame represents the normal sequence and the lower one represents the variant. B: The highlighted zones respectively indicate 13 novel missense variants among 8 species. C: Distribution of genetic cause in our ARCA families ( $n=27$, including two previously reported). 


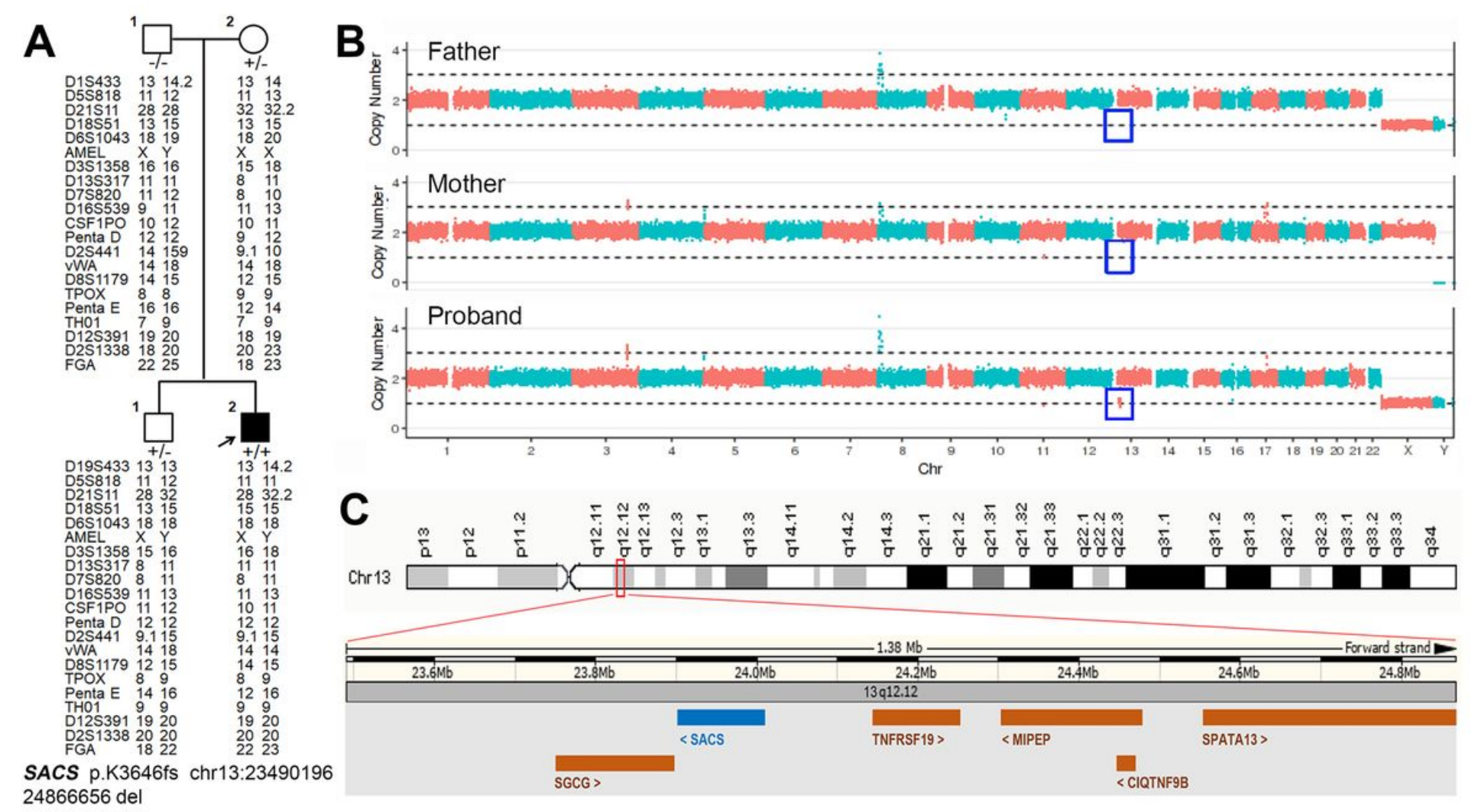

Figure 2

A de novo large deletion in a patient with ARSACS. A: The pedigree of case 1 shows segregation of p.K3646fs in SACS and chr13:23490196-24866656 del. The analysis of the repeat numbers of 21 core STR loci in the four participants showed that the probability of the patient being the alleged parents' biological son is $99.99 \%$. Open symbol: unaffected; filled symbol: affected; square: male; circle: female; arrow: proband of the family. Symbol with " $+/+$ " indicate patient. Symbol with " $+/-$ " or " $-/+$ " indicate mutation carrier. B: CNVseq of the proband and his parents shows that chr13:23490196-24866656 del is a de novo mutation, as indicated by the blue pane. C: Schematic diagram of the known genes in this deletion region (reference human Genome Build GRCh37, UCSC Assembly hg19). 

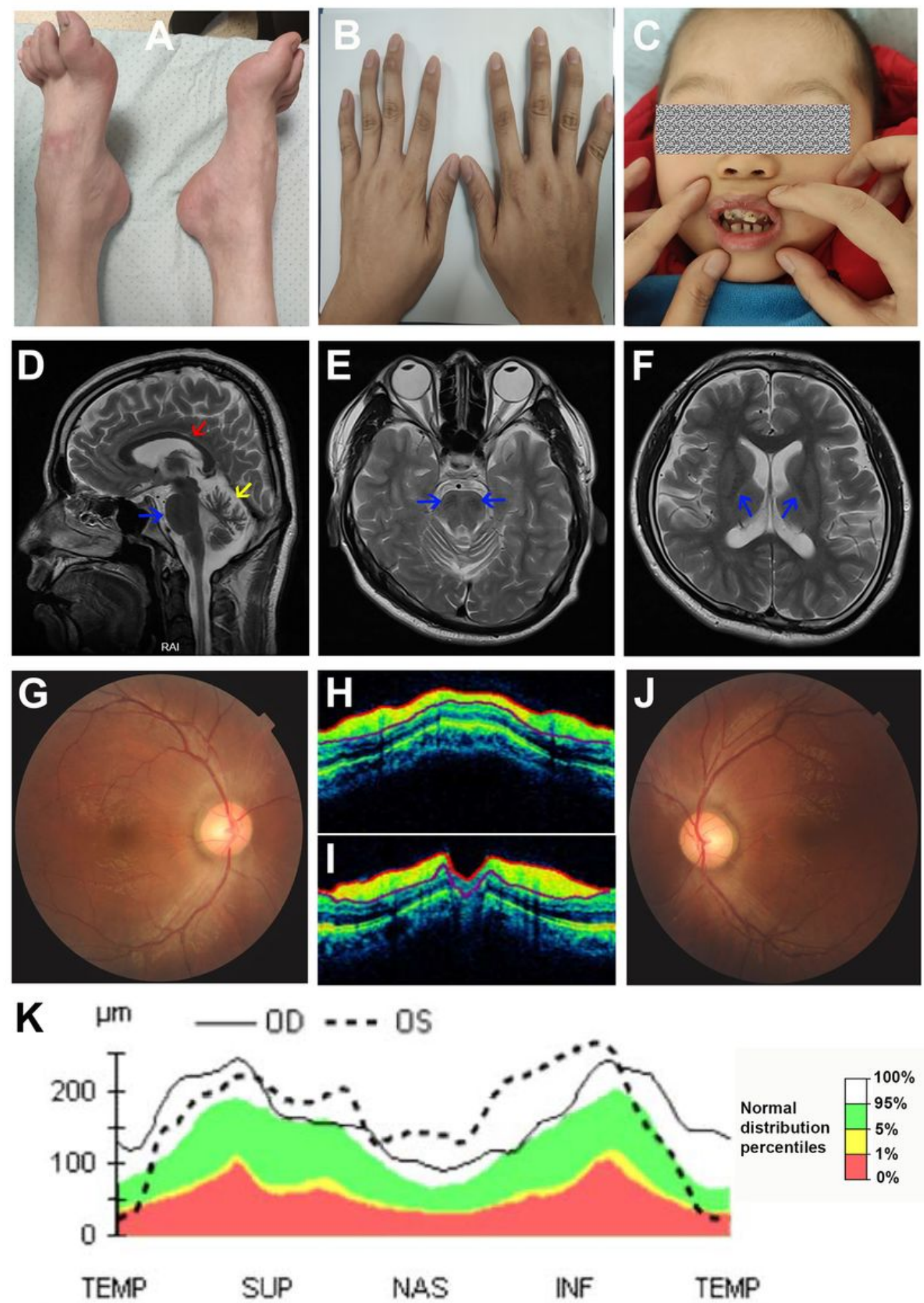

\section{Figure 3}

Clinical features of patients with ARSACS. A-C: The special phenotypes of ARSACS: pes cavus (A), flexion deformity of fingers in case 4 (B) and abnormality of dentition in case 1 (C). D-F: Classic brain MRI in case 2: (D) Sagittal T2 sequence shows thinning of the corpus callosum (red arrow), superior vermian atrophy (yellow arrow), and bulky pons (blue arrow); (E) axial T2 shows bilateral pontine linear hypointense lesions (arrow); (F) axial T2 shows hyperintensities around the thalamus (arrows). G-K: 
Typical retinal findings in case 4: Fundus photographs of the right $(G)$ and left eye $(\mathrm{J})$ show yellow streaks of hypermyelinated fibers radiating from the edges of the optic disc; ocular coherence tomography imaging of the right $(\mathrm{H})$ and left eye $(\mathrm{I})$ show thickened retinal nerve fiber layer (RNFL) (the yellow areas); (K) statistical graph of OCT showing thickening of the RNFL. The black line indicates oculus dexter (OD), that is, the right eye; the dotted line indicates oculus sinister (OS), the left eye. The green band represents the $5 \%$ to $95 \%$ range of the normative data. (Quadrants: TEMP = temporal; SUP = superior; NAS = nasal; INF = inferior).
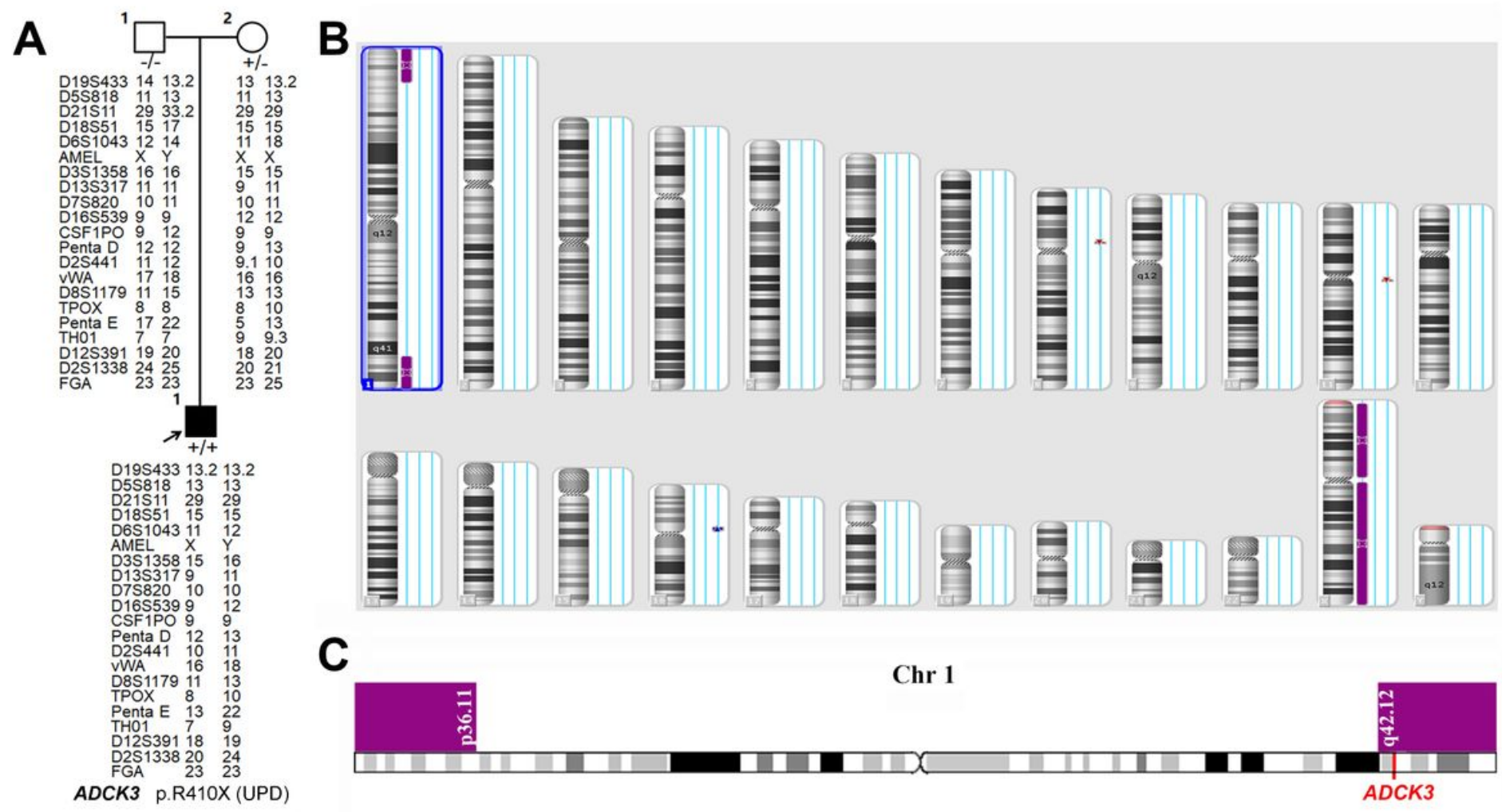

\section{Figure 4}

A maternal uniparental disomy in a patient with SCAR9. A: The pedigree of case 12 shows the segregation of p.R410X in ADCK3. The analysis of the repeat numbers of 21 core STR loci in the three participants showed that the probability of the patient being the alleged parents' biological son is $99.99 \%$. B: Uniparental disomy of chromosomes $1 p$ and $1 q$ detected by Affymetrix CytoScan ${ }^{\circledR}$ Dx Assay in the patient. C: Schematic diagram of ADCK3 in this UPD region (1pterp36.11 and 1q42.12qter; reference human Genome Build GRCh37, UCSC Assembly hg19).

\section{Supplementary Files}

This is a list of supplementary files associated with this preprint. Click to download.

- Additionalfile1SupplementaryFigure1.tif

- Additionalfile2SupplementaryVideo1.mp4 
- Additionalfile3and4SupplementaryTable.xIsx 